\title{
Migration of physician from developing central and eastern Europe to more developed english speaking western nations: What are the factors, causes and potential solutions?
}

J. R. Olechna, D. J. West, Jr.

University of Scranton, Scranton, Pennsylvania

Submitted: 12.8.2015 Revised: 12.1.2016 Accepted: 14.4.2016

\section{Reviewers:}

V. Kremery

St. Elisabeth University of Health and Social Sciences, Bratislava Slovakia

V. Okoth

Adjunct lecturer Catholic University of Eastern Africa

Key words: Migration, Europe, causes, solutions

\begin{abstract}
:
As information and economic growth continues to flourish in the developed nations, these developing nations are beginning to rise to the challenge of globalization. As these nations begin to develop economies of scale and start competing in the world marketplace, there are both positive and negative manifestations. While their policy makers and social sectors become more successful, there is evidence of a "brain-drain" in these developing nations. There are many suggestions as to why this is occurring, such as, but not limited to: economic; and social mobility; gender and race/ethnic disparities; culture clashes; academic integrity and advancement; career development. The issue at hand is not whether or not a "brain drain" is in effect, but to recognize this problem and offer solutions to address it. The purpose of this paper is to come up with potential suggestions and solutions, using evidence based research in relation to the four largest developed English-speaking countries: United Kingdom, Australia, United States, and Canada.
\end{abstract}

\section{Introduction}

The United States with a population of approximately 316.6 million people or roughly $5 \%$ of the world's population employs $11 \%$ of the globe's Physicians - and its demand is growing at an unprecedented rate. Today, $25 \%$ of US Physicians are international medical graduates, and the number is even higher in the UK, Canada, and Australia. These numbers may be shocking, that's because they are. There are significant issues that arise when a country relies heavily on professionals from another. Many of these graduates come from poor countries with high disease burdens - precisely those nations that can least afford to lose their professionals. (Chen, 1850). 
The idea of "brain drain" is not a new issue that has just been unearthed. Brain drain can be defined as the migration of health personnel in search of a better standard of living and quality of life, higher salaries, access to advanced technology, and more stable political conditions in different places worldwide. This trend has led to concerns that the outflow of Healthcare Professionals is adversely affecting the healthcare system in developing countries. Hence, adversely affecting the health of the population.

\section{United States Equivalent}

The United States has its own national equivalent to this global idea of brain drain. Healthcare professionals tend to migrate from less populated rural areas to more densely populated urban or metropolitan areas for various reasons often monetary. The healthcare systems in these rural areas are adversely affected by the migration of Healthcare Professionals, thus the population of the rural areas are adversely affected.

International migration first emerged as a major public health concern in the 1940s when many European professionals emigrated to the UK and USA. In the 1970s, the World Health Organization (WHO) published a detailed 40-country study on the magnitude and flow of the Health Professionals. According to this report, close to $90 \%$ of all migrating Physicians, were moving to just five countries: Australia, Canada, Germany, UK, and USA (Dodani, 487)." This is also an issue that does not seem to be diminishing. The question then is where are these Health Professionals coming from and what can be done to reduce the astronomical statistics.

In 2003, a study by Wendy Hansen from the Maastricht Economic Research Institute on Innovation and Technology (MERIT) found that "the US, Canada and Australia continue to draw talent from the European
Research Area (ERA) (Grigolo, 118)." This is not something that the European Union took lightly and further research was conducted. Upon further investigation it was concluded that the research may have been exaggerated and the results were not as drastic as depicted. This does not mean that this trend is not occurring but not at the rate specified.

\section{Methods}

It is difficult to quantitatively measure Physician emigration on a global scale because there is not a universal protocol in place. Therefore, a variable must be chosen in order to get a better grasp and understanding of how these Physicians are moving throughout developing Central and Eastern Europe and the aforementioned developed English speaking Western Nations. The variable of Physician demographics seemed a fitting method of measurement.

\section{Results}

The following 2009 data was retrieved from the WHO:

It can be seen that in 2009, four of the European nations examined have a higher indication of Physicians per 10,000 population, with the exception of Poland.

The following 2011 data was retrieved from the Organization for Economic Co-operation and Development (OECD):

It can be seen that in 2011, three of the European nations examined have a higher indication of Physicians per 10,000 population, with the exception of Hungary which is lagging behind Australia, and Poland.

When compared side by side, the data shows an intriguing depiction:

It can be seen that when 2009 data is compared with that of 2011 , all of the nations examined, with the exception of the United States, represent an increase in Physicians per 10,000 population. There can 
be many explanations as to why the United States shows a decrease in Physicians per capita such as medical school graduates or, the perpetual aging and utilization of healthcare resources by the baby boomer generation, but that is not within the scope of this paper.

Upon reviewing the literature, measuring the Physician demographic data for 2009 and 2011, and comparing the results side by side, there is no significant evidence that suggests there is a mass exodus of Physicians from Central and Eastern Europe to English speaking Western Nations. Although the data does not prove movement within the aforementioned regions, there may be movement within the European Union. This can be seen as a positive notion by the EU by virtue that their idea of open borders is effective. A trend is seen that Eastern Europeans tend to move into the expanding EU nations. This may give an indication that the EU may face similar problems of brain drain like that of the US in the future.

The next question to ask is if the international medical graduates being employed by the four leading English speaking countries are not from Central and Eastern Europe, where are they coming from. Evidence suggests that many Physicians are mostly migrating from sub-Saharan Afri$\mathrm{ca}$, and Southeast Asia. Countries such as Ghana, Uganda, Sudan, India, Pakistan, and the Philippines are held victim to the brain drain trend.

It is a basic human right for individuals to move freely as they please and to live where they choose. Therefore, it is unethical to force Health Professionals to remain in their native lands. Because developing nations have a higher demand for these professionals, it is unlikely that they will make it more difficult for them to migrate or explore efforts to reduce the influx. Hence, the responsibility lies with the nations that they are migrating from. "Only when health staff, whatever their cadre, have the tools they require to do their job, training opportunities, a network of supportive colleagues, and recognition for the difficult job they do, are they likely to feel motivated to stay put when opportunity beckons from elsewhere (Dodani, 490)." The nations held victim must train, retain, and sustain their professionals.

There are some limitations to this study. Firstly, only research in English was examined. The data presented does not break down the Physicians into specialty or Board Certification. Physician demographics, while an effective variable for this study, may not be the most ideal variable and another must be created in order to measure Physician migration. This study also does not measure movement within the European Union.

\section{Conclusion}

In conclusion, brain drain is a global health concern, but there is not enough evidence that suggests a significant migration of Physicians from developing Central and Eastern Europe to more developed English speaking Western Nations. There is significant evidence upon reviewing the literature that suggests brain drain from Africa and Asia. Source countries must learn to train, retain, and sustain their health professionals. Further research must be conducted globally and especially in the countries suffering from brain drain.

\section{References}

Arah, Onyebuchi A, Uzor C. Ogbu, Chukwadi E. Okeke (2008) Too Poor to Leave, Too Rich to Stay: Developmental and Global Health Correlates of Physician Migration to the United States, Canada, Australia, and the United Kingdom. American Journal of Public Health 98.1 148-54. 
Chen, Lincoln C, M.D., Jo Ivey Boufford, M.D. (2005) Fatal Flows - Doctors on the Move. The New England Journal of Medicine 353.17 1850-52.

Dodani, Sunita, Ronald E. LaPorte (2005) Brain Drain from Developing Countries: How Can Brain Drain be Converted into Wisdom Gain? Journal of the Royal Society of Medicine 98.11 487-91.

Dovlo, Delanyo (2005) Taking More Than a Fair Share? The Migration of Health Professionals from Poor to Rich Countries. PLoS Medicine 2.5 0376-0379. Dwyer, James (2007) What's Wrong with the Global Migration of Health Care Professionals? Individual Rights and International Justice. The Hastings Center Report 36-48.

Grigolo, Michele, Matthieu Lietaert, Ramon Marimon. (2010) Shifting From Academic 'Brain Drain'to 'Brain Gain' in Europe. European Consortium for Political Science 9 118-30.
Loh, Lawrence C, Cesar Ugarte-Gil, Kwame Darko (2013) Private Sector Contributions and their Effect on Physician Emigration in the Developing World. Bull World Health Organ 91 227-33.

The Metrics of the Physician Brain Drain. The New England Journal of Medicine 353.17 (2005): 1810-18.

\section{Corresponding author}

Daniel J. West, Jr. PhD, FACHE

Professor \& Chairman

Department of Health Administration \& Human Resources

Panuska College of Professional Studies

417 McGurrin Hall

Scranton, Pennsylvania 18510-4597 